\title{
LA MASTURBATION AU LABORATOIRE
}

\author{
J.C.Czyba
}

\author{
Département de Gynécologie, Oncologie médicale, Sénologie et Médecine de la Reproduction \\ Hôpital E. Herriot - Place d'Arsonval - 69003 LYON
}

\section{MASTURBATION IN THE LABORATORY:}

The considerable development over the past few years of the infertility investigation of the couple and of the assisted reproductive technics implies that the laboratories of biology of reproduction receive numerous men. These men have to masturbate to produce an ejaculate. The medicalisation of a forbidden act for most cultures does not make it commonplace and may give rise to various difficulties. Key-words : masturbation, sperm, taboo. Andrologie, 1991, 1 : 34-35

Le recueil du sperme pour la réalisation d'un spermogramme, ou d'une quelconque "Procréation médicalement assistée", implique une éjaculation obtenue le plus souvent par masturbation solitaire. La masturbation est dans toutes les civilisations l'objet d'un interdit, explicite ou implicite, mais toujours présent.

La demande du recours à la masturbation constitue une agression. Le plus souvent, la contradiction entre l'interdit et la nécessité médicale d'obtenir le sperme est dépassée dans l'espoir de la vérification ou du rétablissement de la fertilité. Pour certains, cependant, la prédominance de l'interdit entraîne des comportements d'évitement ou une inhibition de l'éjaculation. Nous entendons par comportements d'évitement : le recours au coït interrompu, le recueil de l'éjaculat dans un préservatif spécial (non spermicide), la masturbation par la partenaire (10\% de nos patients viennent au laboratoire accompagnés de leur épouse). Certains médecins confrontés au même interdit ne précisent pas au patient de quelle façon doit être obtenu le sperme, ou préconisent d'emblée une autre méthode que la masturbation, ou préfèrent résolument le test post coïtal au spermogramme.

Le recueil au laboratoire est habituellement exigé pour assurer un meilleur contrôle du délai entre l'émission et l'examen et pour éviter les inconvénients et les incertitudes du recueil à domicile et du transport. La masturbation, solitaire ou assistée, réclame une intimité dont les conditions sont souvent difficiles à assurer dans le cadre d'un laboratoire hospitalier. Le consultant, ou l'homme engagé dans une procréation médicalement assistée, doit franchir quelques étapes éprouvantes avant de parvenir au terme de sa corvée : séjour plus ou moins prolongé dans une salle d'attente où tous les hommes sont supposés venir donner leur sperme ; accueil par une hôtesse qui, avec une froideur toute professionnelle, demande la durée de l'abstinence sexuelle ; masturbation dans une pièce à l'isolement phonique toujours jugé insuffisant ; remise, de la main à la main, du flacon de sperme à l'hôtesse ou à la technicienne (ou pénible aveu de l'impossibilité d'éjaculer). Les plaintes spontanées sont fréquentes et concernent généralement la qualité de l'accueil (manque de discrétion) et l'inconfort des locaux.

Tous les laboratoires de spermiologie ont la notion d'un certain taux d'"échecs", le patient ressortant de la cabine, après un assez long délai, sans avoir pu obtenir une éjaculation. Chez 3.000 consultants venus dans notre service pour la réalisation d'un premier spemogramme, nous avons évalué le taux d'échecs à : $1,7 \% \mathrm{chez}$ 2.275 français, $8 \%$ chez 611 maghrébins et $37 \%$ chez 114 non musulmans d'Afrique noire. Nous avons relevé des taux voisins chez les hommes venus pour une fécondation in vitro ou une insémination artificielle homologue. Dans leur quasi totalité, les français expliquent leur insuccès par les circonstances mêmes du prélèvement: accueil et locaux peu discrets, lieu inconfortable et peu propice à l'éclosion de fantasmes érotiques. Presque tous les nord africains font référence à l'interdit coranique de la masturbation et précisent qu'ils n'y ont jamais eu recours. Les sujets d'Afrique noire ne font état ni d'un interdit socio-culturel, ni de l'inconfort de la situation; ils affirment tout ignorer de la masturbation et ne l'avoir jamais pratiquée. Nous n'entreprendrons pas ici une interprétation des justifications fournies par les uns et les autres pour nous borner au constat de l'existence de l'interdit inhibiteur et de sa rationalisation stéréotypée en fonction de la culture et de l'ethnie.
Un groupe particulier est constitué par les malades (Hodgkin, cancer du testicule...) qui, à la veille d'un traitement stérilisant, tentent de préserver leur fertilité par une cryoconservation du sperme. Il s'agit, le plus souvent, de jeunes hommes pour lesquels le recours à la masturbation s'inscrit dans un contexte somatique et psychologique dramatique. Le taux d'échecs dans notre pratique est de 4\% (14/351 français). Notons qu'aucun de ces patients n'avait été informé par son médecin traitant de la façon dont est obtenue l'éjaculation.

Les "Procréations médicalement assistées" ont fait apparaître la "Procréation sans sexualité". Il serait plus juste de parler de procréation sans coït car la sexualité est loin d'être totalement évacuée de la fécondation in vitro et de l'insémination artificielle. Dans tous les cas, le sperme provient d'une masturbation qui résume la participation du partenaire à l'acte procréateur, mais dont on ne peut dire qu'elle constitue un comportement sans rapport avec la sexualité. De plus, en raison de l'interdit et du caractère honteux de l'acte solitaire, on peut admettre que certains intègrent mal dans leur système de représentations cette nouvelle façon de devenir père. On doit également souligner le paradoxe insolite de la masturbation fécondante.

Les donneurs de sperme pour insémination hétérologue différent des groupes précédents dans la mesure où ils ne sont pas motivés par leur propre problème de fertilité. Ils ne sont pas demandeurs d'aide médicale mais viennent pour rendre service à un couple infertile. Dans notre laboratoire, nous avons remarqué que les donneurs discutent volontiers, avant toute prestation, les conditions dans lesquelles est effectué le recueil et laissent facilement émerger quelques fantasmes en exprimant par exemple leur surprise d'avoir à se livrer à une pratique solitaire. Alors qu'il est clairement établi, lors du premier entretien, que 5 ou 6 dons au moins seront nécessaires pour disposer d'un stock raisonnable de paillettes, $13 \%$ de nos volontaires (24/179 français) sont perdus de vue après le premier don. Déception après la révélation de la non concordance des fantasmes et de la réalité ? 
Le développement des investigations de la fertilité du couple et des procréations médicalement assistées s'accompagne d'une banalisation apparente de la masturbation. En 1990, plus de 7.000 masturbations ont été effectuées dans notre laboratoire (examens de sperme, FIV, IAC, dons de sperme...). Ces actes n'entrent en tant que tels ni dans le discours de l'administration hospitalière ni dans celui des médecins. Nous vérifions constamment la réaction horrifiée de nos confrères à qui nous demandons de nous adresser leurs patients pour vérifier l'effet d'une maladie ou d'une thérapeutique sur le spermogramme. Les justifications médicales autorisent parfois le recours à la masturbation, mais ne lèvent pas les difficultés qu'entraine la persistance de l'interdit. Notons enfin la complète asymétrie entre les méthodes de recueil des gamètes masculins et féminins : d'un coté masturbation, supposée être agréable, objet de plaisanteries et d'interdits culturels ou moraux; de l'autre, ponction chirurgicale des follicules ovariens, toujours désagréable, mais non soumise à interdit.
Résumé : Le développement considérable au cours de ces dernières années de l'investigation de la stérilité du couple et des procréations médicalement assistées implique que les laboratoires de biologie de la reproduction reçoivent un nombre important de patients qui doivent se masturber pour fournir un éjaculat. La médicalisation d'un acte interdit par la plupart des cultures ne le banalise pas dans tous les cas et diverses difficultés peuvent surgir. Mots clés : Masturbation, interdit, sperme. Andrologie : 1991, 1 : $34-35$

\title{
ASPECTS RÉCENTS DE L'EXPLORATION ET DU TRAITEMENT DE L'IMPUISSANCE
}

\author{
Paris, Mardi 15 Octobre 1991. \\ Réunion organisée par la SOCIETE D'ANDROLOGIE DE LANGUE FRANÇAISE \\ Renseignements : J.BUVAT, 49 rue de la Bassée, 59000 LILLE, FRANCE
}

\begin{abstract}
MATINEE : Président : E. WESPES
Données physiologiques et physiopathologiques

P. BONDIL

Facteurs psychologiques et conjugaux : toujours d'actualité

M. BUVAT-HERBAUT

Evaluation objective de la contribution organique : rigidométrie nocturne, stimulation visuelle ou injection intracaverneuse de drogue vasoactive ?

M. SCHOUMAN

Progrès rêcents des explorations vasculaires

C. STIEF

Etude du tissu caverneux : l'évaluation clé de demain

E. WESPES

L'électromyographie du pénis : nouvelle exploration du muscle lisse et de son contrôle neurologique

E. QUITTELIER

Testostérone libre et biodisponible : des critères plus fiables d'androgénicité ?

M. HARTER
\end{abstract}

APRES-MIDI : Président : H. NAVRATIL

Traitements de l'impuissance : le décalage entre l'offre et la demande

J. BUVAT

Où en sont les thérapies psychosexuelles?

J. TIGNOL

Traitement palliatif : injections intracaverneuses ou vacuum?

A. LERICHE

Table ronde : L'apport de la prostaglandine E1 dans l'exploration et le traitement de l'impuissance : Modérateur

M. ETCHEVERRY

- L'expérience d'un pionnier

W. STACKL

- Les autres expériences

J. BUVAT, U. CALVET, A. LEMAIRE, E. WESPES

Etat des traitements chirurgicaux

J.P. SARRAMON 